\title{
DIGITALCOMMONS
}

@WAYNESTATE-

\section{Human Biology}

Volume 81

Issue 2 Special Issue on Demography and Cultural

Article 1

Macroevolution

2009

\section{Introduction: Demography and Cultural Macroevolution}

James Steele

University College London, j.steele@ucl.ac.uk

Stephen Shennan

University College London, tcfasts@ucl.ac.uk

Follow this and additional works at: http://digitalcommons.wayne.edu/humbiol

\section{Recommended Citation}

Steele, James and Shennan, Stephen (2009) "Introduction: Demography and Cultural Macroevolution," Human Biology: Vol. 81: Iss. 2-3, Article 1. Available at: http://digitalcommons.wayne.edu/humbiol/vol81/iss2/1 


\title{
Introduction: Demography and Cultural Macroevolution
}

\begin{abstract}
The papers in this special issue of Human Biology, which derive from a conference sponsored by the Arts and Humanities Research Council (AHRC) Center for the Evolution of Cultural Diversity, lay some of the foundations for an empirical macroevolutionary analysis of cultural dynamics. Our premise here is that cultural dynamics - including the stability of traditions and the rate of origination of new variants - are infl uenced by independently occurring demographic processes (population size, structure, and distribution as these vary over time as a result of changes in rates of fertility, mortality, and migration). The contributors focus on three sets of problems relevant to empirical studies of cultural macroevolution: large-scale reconstruction of past population dynamics from archaeological and genetic data; juxtaposition of models and evidence of cultural dynamics using large-scale archaeological and historical data sets; and juxtaposition of models and evidence of cultural dynamics from large-scale linguistic data sets. In this introduction we outline some of the theoretical and methodological issues and briefl y summarize the individual contributions.
\end{abstract}

\section{Keywords}

cultural transmission, demography, dispersal, carrying capacity, prehistoric Europe, language change.

\section{Cover Page Footnote}

We would like to acknowledge the considerable assistance received from the Human Biology editorial offi ce in preparing this issue; in particular, Franz Manni, Nancy Wise, and Mimi Braverman. The conference was funded by an AHRC Phase Two Research Center grant to the AHRC Centre for the Evolution of Cultural Diversity (http:// www.cecd.ucl.ac.uk). We thank Manu Davies, CECD Administrator, for making the practical arrangements that enabled this conference to take place. 


\title{
Introduction: Demography and Cultural Macroevolution
}

\author{
JAMES STEELE $^{1}$ AND STEPHEN SHENNAN ${ }^{1}$
}

\begin{abstract}
The papers in this special issue of Human Biology, which derive from a conference sponsored by the Arts and Humanities Research Council (AHRC) Center for the Evolution of Cultural Diversity, lay some of the foundations for an empirical macroevolutionary analysis of cultural dynamics. Our premise here is that cultural dynamics-including the stability of traditions and the rate of origination of new variants - are influenced by independently occurring demographic processes (population size, structure, and distribution as these vary over time as a result of changes in rates of fertility, mortality, and migration). The contributors focus on three sets of problems relevant to empirical studies of cultural macroevolution: large-scale reconstruction of past population dynamics from archaeological and genetic data; juxtaposition of models and evidence of cultural dynamics using large-scale archaeological and historical data sets; and juxtaposition of models and evidence of cultural dynamics from large-scale linguistic data sets. In this introduction we outline some of the theoretical and methodological issues and briefly summarize the individual contributions.
\end{abstract}

The papers in this special issue of Human Biology are derived from a conference sponsored by the Arts and Humanities Research Council (AHRC) Center for the Evolution of Cultural Diversity (http://www.cecd.ucl.ac.uk) as part of a series examining the application of evolutionary models in the social sciences. (The AHRC is the U.K. national funding agency for research in these fields. In the past 10 years it has funded a program of interdisciplinary research centers to enable researchers from different institutions and environments to work together on related activities and projects.) The contributions lay some of the foundations for an empirical macroevolutionary analysis of cultural dynamics. Cultural traditions and innovations are socially transmitted between and within generations (by vertical or oblique and horizontal transmission routes, respectively; Cavalli-Sforza and Feldman 1981); learners apply heuristics or rules of thumb to choose when to engage in independent trial-and-error learning and to select which models to copy when this is the preferred strategy (transmission biases; Boyd and Richerson 1985). These processes lead to turnover in cultural traits, which can change the

${ }^{1}$ AHRC Centre for the Evolution of Cultural Diversity, Institute of Archaeology, University College London, 31-34 Gordon Square, London WC1H OPY, United Kingdom.

Human Biology, April-June 2009, v. 81, nos. 2-3, pp. 105-119.

Copyright (C) 2009 Wayne State University Press, Detroit, Michigan 48201-1309

KEY WORDS: CULTURAL TRANSMISSION, DEMOGRAPHY, DISPERSAL, CARRYING CAPACITY, PREHISTORIC EUROPE, LANGUAGE CHANGE. 
selective environment affecting gene frequencies. Adaptive interactions between cultural and genetic evolution have already been well studied in gene-culture coevolutionary theory (Feldman and Laland 1996) and more recently in the niche construction perspective (Odling-Smee et al. 2003).

Our premise here is that cultural dynamics-including the stability of traditions and the rate of origination of new variants-are also influenced by independently occurring demographic processes, such as changes in population size, structure, and distribution over time as a result of changes in rates of fertility, mortality, and migration. Population dynamics will inevitably have an effect on any transmission system in which the level of stability and diversity of traits is dependent on demographic variables. A well-known example in the contemporary world is the reduction in global linguistic diversity and the vulnerability to extinction of languages spoken by small and previously isolated groups; extinction is associated with an increasing scale of sociopolitical integration, higher rates of migration and intermarriage, and the consequent breakdown of intergenerational transmission of the more local language (Barreña et al. 2007; Nettle 1999; cf. Currie and Mace 2009). This accelerated rate of language shift is an example of a macroevolutionary process that is unfolding on a short time scale.

Mesoudi et al. (2006) proposed a multidisciplinary framework for the Darwinian analysis of cultural dynamics and drew an explicit parallel between evolutionary archaeology, cultural anthropology, and comparative anthropology (among the cultural sciences) on the one hand and the macroevolutionary disciplines in biology (paleobiology, biogeography, and systematics, respectively) on the other. Cultural macroevolution refers to the historical processes that explain cultural similarities and differences between human populations (Borgerhoff Mulder et al. 2006).

Historical linguistics should certainly be added to the list of cultural disciplines with a macroevolutionary focus in Mesoudi et al.'s (2006) scheme. There is, of course, a massive literature on the large-scale correlations between genetic and linguistic variation, much of it influenced by the integrative approach of $\mathrm{Ca}$ valli-Sforza and his collaborators, who see the two systems as coevolving as a result of population expansion and splitting, geographical isolation, and parental transmission. Parental transmission is the sole mechanism of genetic inheritance and, as Cavalli-Sforza et al. $(1988,1992)$ would argue, the predominant mechanism of linguistic inheritance in small-scale societies. In prehistoric archaeology, such demographic interpretations of cultural macroevolution are also already familiar, particularly in the much debated farming-language dispersal hypothesis for the spatial spread and diversification of languages, such as those of the Bantu, Austronesian, or Indo-European groups (Diamond and Bellwood 2003). Demographic hypotheses have also been advanced to explain similarities and differences in material culture and social systems when comparing societies, notably in the interpretation of a strong phylogenetic signal in such systems as indicative of a bifurcating population history (with conservative transmission assumed to be characteristic within lineages; e.g., Collard et al. 2006).

The current state of play in phylogenetic analyses of cultural diversity and their demographic interpretation is the subject of a companion collection of papers 
also sponsored by the AHRC Centre for the Evolution of Cultural Diversity (Steele et al. 2010). In this special issue of Human Biology, however, we focus on the reconstruction of past population dynamics and on other examples of cultural macroevolutionary processes that are expected to be responsive to demographic change. We discuss the specific contributions of individual papers in this introduction. Relevant models explored elsewhere include the application of a simple majority voting rule for a binary cultural choice in an admixed population representing two distinct traditions [Ackland et al. (2007) applied this cultural hitchhiking model to language shift in prehistoric farming dispersals]; a vote-with-the-feet model of payoff-biased migration between societies of different initial sizes and with more or less attractive internal norms (Boyd and Richerson 2009); and models of size dependency for cumulative cultural evolution with error-prone intergenerational transmission, where the effective population size must be large enough for pooled individual sampling to extend into the upper tail of a distribution of error effects (Henrich 2004; Powell et al. 2009). For purportedly neutral traits, an example of a model that has been applied to empirical data is the null model of random copying processes (with a population size dependence analogous to that predicted by the neutral model in genetics); this model has been used to explain the empirical increase in diversity in first names in the United States during a period of increasing population size and annual birth rates (Hahn and Bentley 2003).

In some important recent attempts to formulate a macroevolutionary theory of cultural dynamics, investigators adopted models fairly directly from biology, often without much modification, to apply to situations in which the differences that characterize cultural transmission as an inheritance system were also present. These differences include the nonrandom nature of human trial-and-error strategies when exploring a technological design space and the high prevalence of oblique and horizontal transmission, which is biased by inductive heuristics that increase the likelihood of identifying selectively advantageous traits. Although these differences do not invalidate the application of broader Darwinian principles to cultural transmission systems, they do require that new models be developed to explore macroevolutionary dynamics. At present there is a good deal of useful work going on in numerical and simulation modeling of cultural systems to explore the effects of demographic factors on cultural diversity that take into account multiple transmission pathways (e.g., Greenhill et al. 2009; Kandler and Steele 2008; Nunn et al. 2009; Powell et al. 2009). Theory and methods are still evolving, and a number of contributions to this special issue of Human Biology consequently review, develop, and apply different types of models to improve our understanding of cultural macroevolution.

\section{Modeling Cultural Dynamics: Alternative Technical Approaches}

Archaeologists, anthropologists, and linguists formulate theoretical propositions with varying degrees of explicitness and generality, ranging from purely literary models (which simply offer verbal descriptions of a proposed set of causal 
relationships) to analytical models (which derive results from formal analysis of systems of equations). An intermediate level of flexibility and of formal explicitness is represented by numerical and agent-based models, which are more inductive in their approach than fully analytical methods (although typically to differing degrees). Each of these approaches is represented in one or another of the papers in this special issue. We are concerned here with the development and application of explicit and testable theoretical approaches to cultural diversity, so we will not consider further the merits of the literary strategy.

Equations or systems of equations that are modeled analytically are ones that have a characteristic closed-form solution, that is, a solution that can be expressed in terms of well-understood functions and operations. Examples relevant to this special issue include Cavalli-Sforza and Feldman's (1981) analytical models of cultural transmission with varying transmission modes and population structures and Boyd and Richerson's (1985) analytical models of the stability of cultural learning strategies and biased transmission dynamics.

Numerical models, on the other hand, are used to explore mathematical descriptions of relationships for which there is not (or not yet) a recognized closed-form solution. In numerical modeling, equations are solved iteratively over successive time steps and for different parameter constellations to derive empirical generalizations about the behavior of the system, which may then be characterized by diagrams of the phase space (the space of all possible system states). The relationships may be described deterministically (e.g., in a mean field approach, generalizing from a probability distribution of many individual decisions) or stochastically (with a probability distribution being sampled for individual parameter values at each iteration). Numerical models are frequently used to explore the dynamics of complex systems, especially those involving nonlinear interactions among variables. Examples relevant to this special issue of Human Biology include Hahn and Bentley's (2003) and Xu et al.'s (2008) alternative models of the evolution of power-law distributions of traits transmitted by copying and Kandler and Laland's (2009) study of the effects of innovation rate and biased transmission on cultural diversity under selection.

An agent-based model is one in which the state of the system at a given location and time step is determined by a set of local decision rules. These decision rules can be expressed in the form of equations. As Bonabeau (2002: 7280) points out, "A number of researchers think that the alternative to [agent-based modeling] is traditional differential equation modeling; this is wrong, as a set of differential equations, each describing the dynamics of one of the system's constituent units, is an agent-based model." However, many agent-based modelers find it easier to specify their rule sets in terms of logical operators rather than as a full mathematical specification, and this practice may have contributed to the widespread confusion. Agent-based models are particularly useful when individual decision rules are based on discontinuous response functions, are temporally autocorrelated or memory-based, vary with position in a social network, and/or take place in systems that are sensitive to and liable to large random perturbations (Axtell 
2000; Bonabeau 2002). In such cases the system's complexity and its large-scale dynamics are often too great to be tractable by modeling as a system of differential equations. Examples relevant to this special issue of Human Biology include Cox et al.'s (1999) study of the evolution of cooperation in large social groups involving individual heterogeneity, stochasticity, and memory effects, Premo and Hublin's (2009) study of the effects of culturally mediated migration and natural selection on neutral genetic diversity, and Powell et al.'s (2009) study of the effects of group density, population structure, and migration rates on cumulative cultural evolution.

Each of these approaches has its strengths and weaknesses. Analytical derivation of results may require considerable mathematical sophistication and therefore must often be taken on trust by those who wish to apply the results to empirical situations. In addition, there is a limit to the complexity and nonlinearity of the system dynamics that can be explored tractably using such an approach, and that can raise questions about the dependence of results on oversimplifying assumptions about the modeled system. Numerical modeling places large demands on programming skills and can produce more immediately accessible results, but nonetheless underlying technical issues (e.g., concerning the numerical stability of a discretization of a continuum model) must be addressed satisfactorily for the results to be valid. In addition, the application of a more inductive approach may cause analytically derivable results to be overlooked, leading to unduly complicated expositions of the system dynamics (the same applies to agent-based models). Agent-based models are typically more flexibly defined and idiosyncratic and involve higher-dimensional parameter spaces, which enhances their realism; as a consequence, however, their results are vulnerable to misinterpretation (e.g., when artifacts of the rule set or its software implementation are misinterpreted as emergent properties of the modeled system), overfitting (when the model explains the noise as well as the signal in the empirical system being modeled), and lack of robustness (when small variations to parameter constellations produce large changes in outcome). If purely technical weaknesses are resolved by appropriately careful and thorough implementation, then each approach has its own self-evident merits; in fact, we believe that there is a good deal to be said for complementing or contrasting simulation output with appropriate analytical work within a single study (e.g., Galan and Izquierdo 2005; Whitehead and Richerson 2009).

\section{Confronting Models With Data}

The contributors to this special issue of Human Biology focus on three sets of problems relevant to empirical studies of cultural macroevolution: large-scale reconstruction of past population dynamics from archaeological and genetic data (Steele; Ray and Excoffier; Bentley, Layton, and Tehrani; Chamberlain; and Zimmermann, Hilpert, and Wendt), juxtaposition of models and evidence of cultural dynamics using large-scale archaeological and historical data sets (BocquetAppel and Tuffreau; Riede; Shennan; and Richerson, Boyd, and Bettinger), and 
juxtaposition of models and evidence of cultural dynamics from large-scale linguistic data sets (Kandler, and Wichmann and Holman). The appropriate data to juxtapose with Vogt's paper on agent-based modeling of group size and language evolution are those relating to the evolution of hominin grouping strategies in the Paleolithic archaeological record, and some of the issues have been reviewed elsewhere by Buckley and Steele (2002) and Powell et al. (2009).

Inference of cultural microevolutionary processes from macroevolutionary outcomes is difficult, for all the reasons usually associated with inverse problems (e.g., Boyd and Richerson 2008). To assess the fit between a model and a set of data, forward approaches to modeling use the known dynamics of the empirical system to predict outcomes for a given parameter constellation. In inverse problems the outcomes are known to some degree, but the dynamics of the empirical system and the parameter constellation are unknown and must be estimated by reverse engineering. Typically in such situations, difficulties arise when parameter values cannot be reliably estimated from observable data and when it can be shown that alternative models and alternative parameter constellations would yield the same observed macroevolutionary outcomes. In such situations, once the goodness-of-fit of alternative models has been made comparable by application of some information criterion that penalizes extra degrees of freedom, the problem of equifinality might be resolved by treating the assumptions of one preferred model as axiomatically true; but this is unlikely to satisfy those who disagree! A better approach is to attempt to demonstrate the relative robustness of each competing model's assumptions empirically in modern settings where the relevant microscale processes can also be observed. Similarly, problems of empirical parameter estimation for a model that yields several comparably well-fitting solutions are best addressed by refining the empirical analysis and estimation procedure and by constraining the parameter constellation into a plausible range of values based on modern observations in analogous situations.

The inferential issues associated with inverse problems are widely known and have been discussed in historical population genetics (see, e.g., the impact of coalescent theory in phylogeography; Knowles and Maddison 2002). They have also been discussed in historical linguistics (Evans et al. 2006) and in other cultural disciplines (e.g., Kandler and Steele 2009; Nunn et al. 2009). Accurate and precise demographic parameter estimation is clearly essential if we are to estimate the fit of alternative models of cultural diversity in historical and prehistoric instances, and a significant proportion of the papers in this special issue of Human Biology are therefore dedicated to inference of population dynamics from archaeological and genetic data.

\section{Individual Contributions}

The first group of papers in this special issue focuses on human demography during episodes of range expansion and increased interpopulation interaction. Steele's paper is a comprehensive overview of recent differential equation 
models for human dispersal processes and their application to improving our understanding of a variety of long-standing problems in human prehistory, including the spread of farming into Europe and the colonization of the Americas. Steele emphasizes that such models provide a strong framework for understanding the system being modeled, a basis for the principled addition of extra parameters to models when these models clearly do not fit the data, and good approximations for large population cases. Elsewhere Steele has explored some of the archaeological issues associated with inferring demographic parameter values from the archaeological record of past dispersal episodes, particularly given the limited precision and accuracy of archaeological dating techniques, and he has collaborated in empirical investigations to refine these archaeological chronologies. It is beyond the scope of Steele's review to explore the dynamics of cultural diversification associated with spatial range expansion in prehistoric human populations, but suggestive pilot studies have been explored by Nettle (1999) in the field of historical linguistics and by Buchanan and Collard (2007) for stylistic divergence in archaeological stone tool morphology. This is clearly a field with considerable scope for further modeling work.

Present-day genetic data and their spatial analysis have come to play an increasingly important role in identifying the existence of past population dispersals and in developing and testing methods for distinguishing the operation of selection on past populations from the effects of population movement. Ray and Excoffier's review of this field shows the remarkable progress that has been made by the application of new mathematical-statistical methods, such as approximate Bayesian computation, to the increasingly large amounts of genetic data that have become available. One of the main results of this work has been the demonstration of the potential of the surfing phenomenon. Surfing describes a situation in which even initially rare neutral alleles can rise to high frequencies as a result of being on the crest of a wave of expanding population. When surfing occurs, gene distributions that are difficult to distinguish from selection can be produced. Ray and Excoffier's group has also made major contributions to the testing of different hypotheses about the origin of modern humans using spatial modeling of genetic data. This research has resulted in strong support for the recent African origin model. In addition, their modeling approach to the degree of interaction and interbreeding between modern humans and Neanderthals in Europe and between indigenous foragers and first farmers in Europe has led in both cases to the conclusion that interaction was probably minimal. After outlining this work, Ray and Excoffier go on to identify a series of topics where further progress can be made by integrating the modeling of genetic and archaeological data; the archaeological data can provide informative prior information for the building of Bayesian models.

Bentley, Layton, and Tehrani deal with similar themes to the first two papers, but their contribution is focused on the need to take more account of the ethnographic data on kinship patterns than is usually done when building mathematical models of dispersals and their genetic consequences. In particular, they emphasize the importance of modeling the movements of men and women separately. One 


\section{2 / STEELE AND SHENNAN}

element of this model involves taking into account the implications of matrilocality and patrilocality, which have different effects on male and female movement even when populations are in equilibrium. The effects of matrilocality and patrilocality need to be distinguished from what is going on in dispersal situations, where there may be interactions between indigenous and invasive populations. Such interaction situations are generally associated with introgression of male genes into the indigenous population and, to a lesser extent, of female genes into the invasive population. The reasons for this can be many, but an important one is often hypergyny, the tendency of women to marry up in terms of social and economic status. Hypergyny is important in the context of indigenous-incomer interactions because the incomers are often perceived as being of higher status. Bentley and colleagues review a broad range of ethnographic data and show that in some cases long-standing symbiotic relationships between farmers and foragers on either side of a frontier may exist for ecological reasons. They also illustrate the sometimes massively deleterious effect of such interactions between, for example, European incomers and Aborigines in Australia. Thomas et al.'s (2006) apartheid model is one example of the kind of work that Bentley and co-workers argue is needed, but they emphasize the need to explore a range of sex-specific models that may be relevant to different indigenous-incomer interactions.

Like Bentley et al., Kandler addresses the issue of interactions between populations, but her interest is in their outcomes in the domain of language, with a specific concern for the currently rapid process of language extinction and what might be done to prevent it. Kandler's approach to these issues is similar to that of Steele and involves the use of differential equation systems to model the process of language competition, especially the factors that affect the possibility of linguistic coexistence, the extinction of one language in a two-language system, and the importance of bilingualism. As Kandler shows, despite the fact that many linguists, like archaeologists in their domain, are skeptical of mathematical models, her work is part of a rapidly developing tradition of modeling language competition, and this research is producing important insights that have potential practical applications in language preservation programs. Her own model advances previous work in the field by exploring the dynamics of competition in a system with two monolingual subpopulations and one bilingual subpopulation and processes of vertical transmission that reflect demographic factors and horizontal transmission that is influenced by sociolinguistic factors. One counterintuitive result that emerges is that in some circumstances the higher prestige language can be the one that goes extinct.

The next group of papers relates in different ways to the effects of population size on cultural dynamics. Richerson, Boyd, and Bettinger's paper in effect outlines a general theory of demography and rates of innovation and, in particular, the factors that affect carrying capacities. It is difficult to exaggerate the importance of their starting point, which emphasizes the general validity of MalthusioDarwinian theory for an understanding of the relation between demography and cultural change. Like the rest of the natural world, human capacities for increase 
quickly outstrip the potential of the environment to support the resulting numbers. Relative to the time scales that archaeologists deal with, the time to reach carrying capacity, even from a minimal starting point, is rapid: less than 1,000 years. Thus the key to understanding human population levels is not population pressure but those factors that affect carrying capacities: climate and environment on the one hand and technological innovation leading to increased production per unit time or per unit area on the other. Furthermore, environmental change can lead to decreased carrying capacities as well as to increased ones, whereas technological changes can have the same effect if new technologies are not sustainable and lead to the overexploitation of resources that cannot be replaced to maintain or increase the carrying capacity. Like Powell et al. (2009), Richerson, Boyd, and Bettinger point to the link between small populations, which may be environmentally limited, and low innovation rates and cultural devolution, but they see this link as the first of three successive stages in the relationship between technology and population. The last stage, the Industrial Revolution, was characterized by a rate of technological innovation that could keep pace with the intrinsic rate of increase in human populations.

Vogt reviews different styles of modeling language evolution and the potential relevance of demographic effects to how language as a complex communication system may have evolved, an area that has seen a great deal of modeling work in recent years. While acknowledging that all the different modeling approaches have their uses, Vogt gives most attention to agent-based cognitive models, which are at least an order of magnitude greater in complexity than those used in the modeling of other domains of culture, because of the enormous number of variables that must be included to give values to individual cognitive states. As with the other papers in this special issue of Human Biology and their varying domains, it emerges that population sizes matter in language evolution; for example, compositionality, in which different linguistic elements are put together to refer to different parts of a meaning as opposed to having a single word for each different meaning, is more likely to emerge in larger populations than in smaller ones. The incorporation of real demographic data into models of the evolution of language is some way in the future, but this is really no different from most domains of cultural evolutionary modeling.

In contrast to Vogt's focus on models of the evolution of language as such, Wichmann and Holman address the relationship between population size and rates of change in real languages. This relationship has been a topic of major interest and exploration for the last decade. Wichmann and Holman focus particularly on the hypothesis that rates of change should be greater in languages with smaller numbers of speakers than in larger ones. They take a major step forward by using a recent comprehensive data set of word cognates to create a standardized measure of distance between different languages. They address the question of whether languages with smaller numbers of speakers have diverged further from their common ancestors than languages with larger numbers of speakers. 


\section{4 / STEELE AND SHENNAN}

They find only a small effect, which is far outweighed by other factors. Wichmann and Holman's proposal, based on previous simulations of the propagation of linguistic change on networks, is that the observed pattern of present-day language diversification has arisen because "changes propagate at a local level in a type of network where the individuals have different degrees of connectivity." This proposal bears some similarity to the arguments of Powell et al. (2009) about the factors affecting cumulative cultural evolution. Powell and colleagues showed that migration rates (i.e., degrees of connectivity) were just as important, if not more so, than simple population density. In fact, increased connectivity in itself produced greater effective population sizes and variations in connectivity that did not necessarily correlate with absolute regional population sizes. This finding raises interesting questions about what it is about demographic patterns that relates to culture change. The case of population dispersals and range expansions is clearly not the same as that of the extent of connectivity under conditions of rough population equilibrium.

The final group of papers relates to the estimation of prehistoric demographic patterns and their relationship with empirical cultural evolutionary processes. A key requirement for the testing of models of the relationship between demography and cultural change is the availability of reliable information about past population patterns. Archaeologists and paleodemographers have been extensively debating this topic for many years, and Chamberlain reviews the current state of play with regard to the kinds of inferences that can be made from different lines of evidence. One major area of advance has been the ability to infer the time of the appearance of the modern human life history pattern (150,000 years ago) on the basis of dental information. Another development has been the ability to infer past fertility and mortality patterns on the basis of the distribution of ages at death in human skeletons recovered from archaeological sites. This subject went into something of a crisis in the early 1980s when Bocquet-Appel and Masset (1982) demonstrated that many of the patterns that had hitherto been inferred depended on the modern reference population used in the reconstruction. Recent developments, especially in the application of Bayesian statistical methods, have shown that progress can be made and, in particular, that the generally assumed attritional mortality models in many cases do not actually fit, pointing to the conclusion that skeletal assemblages often derive from catastrophic mortality events of one kind or another. This may tie in with theoretical and ethnographically based arguments that human populations have been subject to regular patterns of boom and bust and that, with other lines of archaeological evidence, especially radiocarbon date distributions, many regions were subject to major population fluctuations [see also Gamble et al. (2005) and Shennan and Edinborough (2007) and the papers by Riede and Shennan in this special issue].

The remaining four papers are concerned with specific case studies relating to the reconstruction of population patterns in prehistory and/or the causes and consequences of these patterns. Bocquet-Appel and Tuffreau look at the extent 
to which technological innovation and adaptive responsiveness to changing climatic patterns, as measured by changing diversity in lithic assemblages, could have affected the carrying capacity of Neanderthal populations during the late Pleistocene of Europe. Like other investigators, they emphasize the importance of population size as a factor affecting innovation rates.

Riede presents a powerful general argument using a range of evidence for the high probability of climatically related demographic fluctuations in huntergatherer populations, especially those living at high latitudes, and for the severe consequences of such fluctuations. He also reviews the increasingly prevalent use of calibrated radiocarbon date distributions as population proxies. The core of the paper, though, is a series of case studies used to look for evidence of population fluctuations and their causes and consequences in the northwest European late Paleolithic and Mesolithic. Riede suggests that the Irish Mesolithic is a classic example of the model proposed by Henrich (2004) to account for the loss of cultural complexity in Tasmania; a complex technology is lost and replaced by a much simpler one at precisely the time that radiocarbon date distributions suggest a population decrease associated with a climatic impact. Riede also suggests that a similar process was at work in the disappearance of the late Paleolithic Hamburgian assemblage in northern Germany and its replacement by the Ahrensburgian; the specific details are different, though, because in the Ahrensburgian we are dealing with a population made vulnerable by being on the fringe of the pioneer recolonization of northern Europe at the end of the last ice age. Riede's third case study demonstrates the potential significance of catastrophic events, such as volcanic eruptions, for understanding regional culture change by showing how the Laacher See volcanic eruption about 13,000 years ago disrupted hunter-gatherer populations and their cultures in northern Germany.

Shennan's paper follows up on similar issues but with a focus on the European Neolithic. Shennan shows how the demographic and social patterns now being documented from the archaeological record can be understood from the point of view of different interrelated aspects of evolutionary theory, including life history theory, population ecology, and reproductive skew theory. Like Riede, Shennan emphasizes that busts as well as booms in regional demographic patterns are visible in the archaeological record of radiocarbon date distributions and other phenomena. And when we take these into account, we come to conclusions that are different from many of the standard views. Thus in central Europe and many other areas it is highly unlikely that Mesolithic populations had a major role in the introduction of farming because they were at historically low levels, most probably because of the drop in available plant and animal production resulting from Holocene forest cover. Equally, it seems that there was a decline in early farming populations in many areas, especially in west-central Europe 400-500 years after farming first appeared, which contradicts the idea that farmers and farming had an increasingly disruptive effect on forager populations, forcing them to switch to farming. 


\section{6 / STEELE AND SHENNAN}

Finally, Zimmermann, Hilpert, and Wendt present a reconstruction of changing population densities in the area of present-day Germany that sets new standards of rigor both in its analytical method and in the way it differentiates between high-density and low-density areas of settlement, with implications for overall regional population estimates. Failure to differentiate these areas has led to consistent overestimation of population densities in most past periods. Like Riede and Shennan, Zimmermann and colleagues point out that the consistently low levels of prehistoric population density seem to have led to instability and regional fluctuations, although the specific reasons for individual fluctuations are hard to discern. However, the fact that these low population densities lasted for millennia after the arrival of farming raises interesting questions about precisely what it was that kept carrying capacities so consistently low. Certainly any link between population density and the degree of political organization cannot be straightforward, because the scale of political organization seems to increase by well over two orders of magnitude between the early and middle Neolithic, without any corresponding population increase. Zimmermann and co-workers' rigorous approach brings out such questions all the more starkly because of the confidence we can have in the patterns they are claiming.

Renfrew contributes a brief epilogue with some thoughts on the themes of this special issue that draw on his extensive experience in integrating the fields of archaeology, linguistics, and genetics. His own contributions to cultural macroevolutionary theory, notably his refutations of hyperdiffusionist explanations of prehistoric cultural patterns in the 1970s and his later work on the farminglanguage dispersal theory of Indo-European origins, were hugely influential for archaeologists interested in these broader questions (e.g., Renfrew 1973, 1987); it is therefore fitting that his comments should conclude this special issue.

Acknowledgments We would like to acknowledge the considerable assistance received from the Human Biology editorial office in preparing this issue; in particular, Franz Manni, Nancy Wise, and Mimi Braverman. The conference was funded by an AHRC Phase Two Research Center grant to the AHRC Centre for the Evolution of Cultural Diversity (http:// www.cecd.ucl.ac.uk). We thank Manu Davies, CECD Administrator, for making the practical arrangements that enabled this conference to take place.

Accepted for publication 4 July 2009.

\section{Literature Cited}

Ackland, G. J., M. Signitzer, K. Stratford et al. 2007. Cultural hitchhiking on the wave of advance of beneficial technologies. Proc. Natl. Acad. Sci. USA 104:8714-8719.

Axtell, R. 2000. Why Agents? On the Varied Motivations for Agent Computing in the Social Sciences. CSED Working Paper 17. Washington, DC: Brookings Institution.

Barreña, A., E. Amorrortu, A. Ortega et al. 2007. Does the number of speakers of a language determine its fate? Int. J. Sociol. Lang. 186:125-139. 
Bocquet-Appel, J.-P., and C. Masset. 1982. Farewell to paleodemography. J. Hum. Evol. 11:321-333. Bonabeau, E. 2002. Agent-based modeling: Methods and techniques for simulating human systems. Proc. Natl. Acad. Sci. USA 99(supp. 3):7280-7297.

Borgerhoff Mulder, M., C. L. Nunn, and M. C. Towner. 2006. Macroevolutionary studies of cultural trait transmission. Evol. Anthropol. 15:52-64.

Boyd, R., and P. J. Richerson. 1985. Culture and the Evolutionary Process. Chicago: Chicago University Press.

Boyd, R., and P. J. Richerson. 2008. Response to our critics. Biol. Philos. 23:301-315.

Boyd, R., and P. J. Richerson. 2009. Voting with your feet: Payoff biased migration and the evolution of group beneficial behavior. J. Theor. Biol. 257:331-339.

Buchanan, B., and M. Collard. 2007. Investigating the peopling of North America through cladistic analyses of early Paleo-Indian projectile points. J. Anthropol. Archaeol. 26:366-393.

Buckley, C., and J. Steele. 2002. Evolutionary ecology of spoken language: Coevolutionary hypotheses are testable. World Archaeol. 34:26-46.

Cavalli-Sforza, L. L., and M. W. Feldman. 1981. Cultural Transmission and Evolution: A Quantitative Approach. Princeton, NJ: Princeton University Press.

Cavalli-Sforza, L. L., E. Minch, and J. Mountain. 1992. Coevolution of genes and languages revisited. Proc. Natl. Acad. Sci. USA 89:5620-5624.

Cavalli-Sforza, L. L., A. Piazza, P. Menozzi et al. 1988. Reconstruction of human evolution: Bringing together genetic, archaeological, and linguistic data. Proc. Natl. Acad. Sci. USA 85:60026006.

Collard, M., S. J. Shennan, and J. Tehrani. 2006. Branching, blending, and the evolution of cultural similarities and differences among human populations. Evol. Hum. Behav. 27:169-184.

Cox, S. J., T. J. Sluckin, and J. Steele. 1999. Group size, memory, and interaction rate in the evolution of cooperation. Curr. Anthropol. 40:369-376.

Currie, T. E., and R. Mace. 2009. Political complexity predicts the spread of ethnolinguistic groups. Proc. Natl. Acad. Sci. USA 106:7339-7344.

Diamond, J., and P. Bellwood. 2003. Farmers and their languages: The first expansions. Science 300:597-603.

Evans, S. N., D. Ringe, and T. Warnow. 2006. Inference of divergence times as a statistical inverse problem. In Phylogenetic Methods and the Prehistory of Languages, P. Forster and C. Renfrew, eds. Cambridge, U.K.: McDonald Institute, 119-129.

Feldman, M. W., and K. N. Laland. 1996. Gene-culture coevolutionary theory. Tr. Ecol. Evol. 11:453457.

Galan, J. M., and L. R. Izquierdo. 2005. Appearances can be deceiving: Lessons learned re-implementing Axelrod's "evolutionary approach to norms." J. Artific. Soc. Soc. Simul. 8(3). http://jasss .soc.surrey.ac.uk/8/3/2.html.

Gamble, C., W. Davies, P. Pettitt et al. 2005. The archaeological and genetic foundations of the European population during the late glacial: Implications for "agricultural thinking." Cambridge Archaeol. J. 15:193-223.

Greenhill, S. J., T. E. Currie, and R. D. Gray. 2009. Does horizontal transmission invalidate cultural phylogenies? Proc. R. Soc. Lond. B 276:2299-2306.

Hahn, M. W., and R. A. Bentley. 2003. Drift as a mechanism for cultural change: An example from baby names. Proc. R. Soc. Lond. B 270:S120-S123.

Henrich, J. 2004. Demography and cultural evolution: Why adaptive cultural processes produced maladaptive losses in Tasmania. Am. Antiq. 69(2):197-221.

Kandler, A., and K. N. Laland. 2009. An investigation of the relationship between innovation and cultural diversity. Theor. Popul. Biol. 76:59-67.

Kandler, A., and J. Steele. 2008. Ecological models of language competition. Biol. Theor. 3:164173.

Kandler, A., and J. Steele. 2009. Innovation diffusion in time and space: Effects of social information and of income inequality. Diffusion Fund. (in press). 


\section{8 / STEELE AND SHENNAN}

Knowles, L. L., and W. P. Maddison. 2002. Statistical phylogeography. Mol. Ecol. 11:2623-2635.

Mesoudi, A., A. Whiten, and K. N. Laland. 2006. Towards a unified science of cultural evolution. Behav. Brain Sci. 29:329-383.

Nettle, D. 1999. Linguistic diversity of the Americas can be reconciled with a recent colonization. Proc. Natl. Acad. Sci. USA 96:3325-3329.

Nunn, C. L., P. H. Thrall, and C. Boesch. 2009. Do transmission mechanisms or social systems drive cultural dynamics in socially structured populations? Animal Behav. 77:1515-1524.

Odling-Smee, F. J., K. N. Laland, and M. W. Feldman. 2003. Niche Construction: The Neglected Process in Evolution. Princeton, NJ: Princeton University Press.

Powell, A., S. Shennan, and M. Thomas. 2009. Late Pleistocene demography and the appearance of modern human behavior. Science 324:1298-1301.

Premo, L. S., and J.-J. Hublin. 2009. Culture, population structure, and low genetic diversity in Pleistocene hominins. Proc. Natl. Acad. Sci. USA 106(1):33-37.

Renfrew, A. C. 1973. Before Civilization: The Radiocarbon Revolution and Prehistoric Europe. London: Jonathan Cape.

Renfrew, A. C. 1987. Archaeology and Language: The Puzzle of the Indo-European Origins. London: Jonathan Cape.

Shennan, S. J., and K. Edinborough. 2007. Prehistoric population history: From the late glacial to the late Neolithic in central and northern Europe. J. Archaeol. Sci. 34:1339-1345.

Steele, J., P. Jordan, and E. Cochrane, eds. 2010. Cultural and linguistic diversity. Special Issue of Phil. Trans. R. Soc. Lond. B (in preparation).

Thomas, M. G., M. P. Stumpf, and H. Harke. 2006. Evidence for an apartheid-like social structure in early Anglo-Saxon England. Proc. R. Soc. Lond. B 273:2651-2657.

Whitehead, H., and P. J. Richerson. 2009. The evolution of conformist social learning can cause population collapse in realistically variable environments. Evol. Hum. Behav. 30:261-273.

Xu, J., F. Reali, and T. L. Griffiths. 2008. A formal analysis of cultural evolution by replacement. In Proceedings of the 30th Annual Conference of the Cognitive Science Society, V. Sloutsky, B. Love, and K. McRae, eds. Austin, TX: Cognitive Science Society. http://cocosci.berkeley .edu/tom/papers/replacement.pdf. 